\title{
A RARE CASE OF MOBIUS SYNDROME ASSOCIATED WITH VENTRICULAR SEPTAL DEFECT AND AORTIC REGURGITATION
}

\author{
Lokesh $\mathrm{S}^{1}$, Shashidhar $\mathrm{G}^{2}$
}

\section{HOW TO CITE THIS ARTICLE:}

Lokesh S, Shashidhar G. "A Rare Case of Mobius Syndrome associated with Ventricular Septal Defect and Aortic Regurgitation". Journal of Evolution of Medical and Dental Sciences 2014; Vol. 3, Issue 31, July 31;

Page: 8670-8673, DOI: $10.14260 /$ jemds/2014/3104

\begin{abstract}
Mobius syndrome is defined in most studies as congenital facial weakness associated with abnormal ocular abduction. Facial nerve and Abducens nerve are most frequently involved, but other cranial nerves may be involved as well. In one third or more of patients, Mobius syndrome can also be associated with musculoskeletal abnormalities like talipes equino varus, brachydactyly, syndactyly, smallness of limbs etc. Mobius syndrome is a very rare condition. In 2007, Mobius syndrome foundation estimated that 2000 individuals worldwide have this condition. Mobius syndrome associated with cardiovascular anomalies are rarely described. Till date four such cases has been reported to the best of our knowledge. Hereby we are reporting a case of mobius syndrome associated with ventricular septal defect and aortic regurgitation.
\end{abstract}

KEYWORDS: Mobius syndrome, ventricular septal defect, aortic regurgitation.

INTRODUCTION: The definition and diagnostic criteria for mobius syndrome vary among authors. Mobius syndrome is basically defined as the congenital disorder involving multiple cranial nerves mainly VIth and VII th cranial nerves. When Mobius syndrome was initially described by Von graefe and Paul julius Mobius only bilateral facial nerve and bilateral abducent nerve involvement along with other features were considered. However Henderson in the year 1939 included even the unilateral facial nerve involvement with bilateral abducent nerve involvement into the definition of Mobius syndrome which is the feature of our case also.

Mobius syndrome can also involve other cranial nerves like IX, X, XI and XII nerves rarely along with various musculoskeletal and cardiovascular anomalies.

CASE REPORT: A 25years old female patient hailing from Bellary presented to medicine OPD with episodes of palpitation since childhood, exertional breathlessness since 4 years and chest pain since last 15 days. She was a $10^{\text {th }}$ sibling for her parents born as home delivery without antenatal checkups with no similar complaints in the family.

On examination she was found to be conscious, cooperative moderately built and nourished her pulse rate was 90 beats per minute regular high volume and it was collapsing in character. Her blood pressure was 140/70 mm of $\mathrm{Hg}$ in right upper limb in supine position and 170/70 mm of $\mathrm{Hg}$ in right lower limb. Respiratory rate was 18 cycles per minute. On head to toe examination she had deformed incissor teeth and pectus excavatum.

On systemic examination cardiovascular system showed systolic thrill in left $2^{\text {nd }} 3^{\text {rd }}$ and $4^{\text {th }}$ parasternal region and harsh pansystolic murmur in same areas on auscultation which was non radiating and early diatolic murmur in erb's area.

CNS examination revealed bilateral abducens nerve palsy and left sided VII nerve LMN type of palsy (Fig.1) 
Her investigations showed RBS-105 mg\% blood urea-18.2 mg\% serum creatinine $-0.7 \mathrm{mg} \%$. Hb-11.4 g\%, TC-10600/cumm, ESR 20mm after 1hour.chest X- ray was showing cardiomegaly of left ventricular configuration. ECG was showing left ventricular hypertrophy.

2D echocardiography revealed situs solitus, dilated left ventricle, $7.4 \mathrm{~mm}$ subaortic ventricular septal defect with left to right shunt, Prolapse of right posterior cusp of aortic valve through VSD, moderate aortic regurgitation, left ventricular dysfunction with ejection fraction of $40 \%$, No vegetation or clot suggestive of ventricular septal defect with aortic regurgitation (Fig.2).

Mobius syndrome is diagnosed based on left side LMN facial nerve palsy with bilateral abducence nerve palsy and associated chest wall and teeth deformity.

CT scan and MRI brain could not be done as the patient was not affordable.

DISCUSSION: Mobius syndrome involves clinical spectrum ranging from unilateral facial nerve paralysis with bilateral abducent nerve paralysis to bilateral facial and abducent nerve paralysis. Even though VIth and VII th nerve involvement is the hall mark of Mobius syndrome, it has got wide spectrum of other clinical features which can be associated less commonly. ${ }^{1}$

Various other names for the mobius syndrome are congenital facial diplegia, nuclear agenesis, congenital nuclear hypoplasia, congenital oculofacial paralysis, and congenital abducens-facial paralysis. $^{2}$

Pathophysiology behind the development of mobius syndrome remains elusive. There are various theories to explain the primary underlying pathogenesis. Most of the cases of Mobius syndrome are sporadic. However few of the cases have either genetic basis or hypoxic injury or intrauterine toxin exposure. Bavinck and weaver proposed that the interruption of the development of subclavian artery and its branches at or around sixth intrauterine week as the cause. ${ }^{3}$

Verzijl and coworker performed electrophysiological studies on 11 patients with mobius syndrome and concluded that Mobius syndrome is not a primary developmental disorder of the facial musculature but is a disorder of the rhombencephalon including both motor nuclei and transversing long tracts. ${ }^{4}$

Pape and pickering in 1972 proposed vascular pathogenesis of Mobius syndrome. Other proposed theories are mesodermal dysplasia of Ist and IInd branchial arches. Benzodiazepenes, coccaine and misoprostol has also been implicated as the cause of Mobius syndrome.

Various classification systems have been proposed for Mobius syndrome. One of those is as follows which is proposed in the year 1979 by Towfighi et al. Group I - Simple hypoplasia or atrophy of CN nuclei. Group II - Primary lesions in peripheral CNS. Group III - Focal necrosis in brainstem nuclei and Group IV - Primary myopathy with no central nervous system (CNS) or cranial nerve lesions. ${ }^{5}$

Clinical Features: Mobius syndrome is usually detected soon after the birth with the features of incomplete closure of the eyelids during sleep, drooling of saliva and difficulty in sucking. However as the child grows up it can be noted that the child is not smiling or not moving its facial muscles on crying. Paralysis of abducent nerve characterized by inability to abduct the eye is frequently associated anomaly and is usually bilateral. Other cranial nerves less frequently involved are oculomotor, trigeminal and hypoglossal nerve resulting in conjugate gaze palsy, paralysis and hypoplasia of the tongue. 
Mild mental retardation may be a feature in $10-15 \%$ of the patient and autism behavior may be seen in 30-40\% of the patient. However many authors report that without formal testing for the intelligence it may be underestimated because of facial appearances. Mobius syndrome is also associated with the limb malformations like club foot, syndactyly, brachydactyly and craniofacial abnormalities like small palpable fissure, hypertelorism, external ear defects, micrognathia less frequently. 6

Cardiac anomalies are very rarely seen in mobius syndrome. Till now to the best of our knowledge only 4 cases are reported with cardiac anomalies in association with Mobius syndrome.

They are mobius syndrome associated with plain dextrocardia, dextrocardia with ventricular septal defect, transposition of great vessels and ventricular septal defect. Our patient had ventricular septal defect with aortic regurgitation which may be the first report. Most of the case reports pertaining to Mobius syndrome are in infants or in children. Only few cases have been reported in adults. Kiratli. H, Erdener $U$ et al has reported a case of Poland-mobius syndrome and acquired progressive bilateral paralytic lower eyelid ectropion in adult patient of 24years age. ${ }^{7}$

Treatment mainly involves supportive and in accordance with symptoms. Special feeding tubes or feeding bottles are needed to maintain the nutrition. Plastic reconstructive surgeries may be beneficial in some individuals with muscle transplantation which is ideally done just before they reach school age. ${ }^{8}$

CONCLUSION: The main purpose of reporting this case is to create awareness among the physicians as it occurs so infrequently that many of the cases go undiagnosed for years as it happened in our case. Because of lack of awareness research towards the potential treatment and cures of Mobius syndrome is also hindered.

\section{REFERENCES:}

1. Douglas. E. mattox. Clinical disorders of the facial nerve. In: Paul W. Flint, Bruce H. Haughey, Valerie J. Lund, John K. Niparko, Mark A. Richardson, K. Thomas Robbins, and J. Regan Thomas (eds). Cummings otolaryngology-head and neck surgery, 5thed; Mosby Elsevier; 2010. p2397.

2. Jones KL. In: Smith DW, ed. Recognizable patterns of human malformations. 4th ed. Philadelphia: Saunders, 1988.p192-193,588-589.

3. Bavinck JN, Weaver DD. Subclavian artery supply disruption sequence: hypothesis of vascular etiology for Poland, Klippel Feil and Moebius anomalies. Am J Med Genet 1986; 23: 903-918.

4. Verzijl HT, van der Z waag B, Cruysberg JR et al. Mobius syndrome redefined: a syndrome of rhombencephalic mal development. Neurology 2003;61:327-333

5. Towfighi J, Marks K, Palmer E, Vannucci R. Möbius syndrome. Neuropathologic observations. Acta Neuropathol (Berl). Oct 1979; 48 (1): 11-7.

6. Kumar D. Moebius Syndrome. J Med Genet 1990; 27: 122-126.

7. Kiratli. H, Erdener. U. Poland-Moebius syndrome: Jpn J Ophthalmol 2000 Nov-Dec; 44 (6): 679-82.

8. Zuker RM, Goldberg CS, Manktelow RT. Facial animation in children with Mobius syndrome after segmental gracilius muscle transplant. Plast Reconstr Surg 2000; 106: 1-8. 


\section{CASE REPORT}

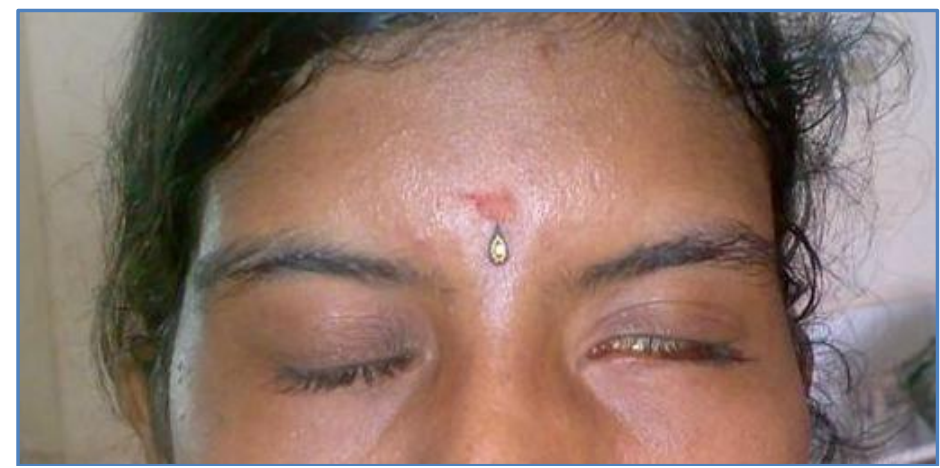

Figure 1

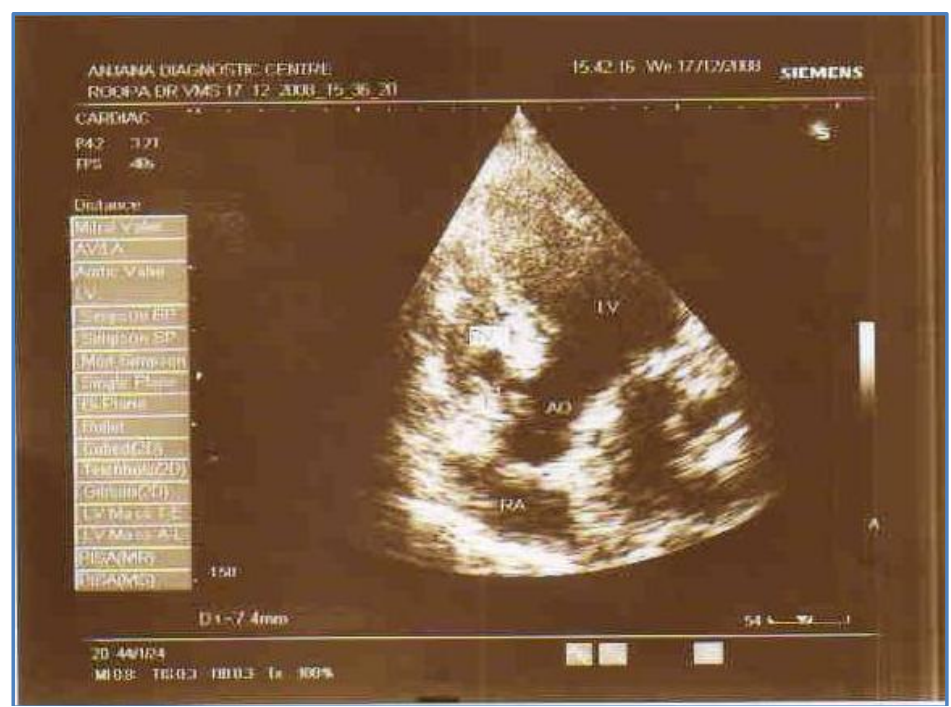

Figure 2

\section{AUTHORS:}

1. Lokesh S.

2. Shashidhar G.

\section{PARTICULARS OF CONTRIBUTORS:}

1. Assistant Professor, Department of General Medicine, Vydehi Institute of Medical Sciences and Research Centre, Whitefield, Bangalore.

2. Assistant Professor, Department of General Medicine, MVJ Medical College and Research Centre, Hoskote, Bangalore.

\section{NAME ADDRESS EMAIL ID OF THE} CORRESPONDING AUTHOR:

Dr. Lokesh S,

No. A-19,

Vydehi Staff Quarters,

A-Block, Vydehi Hospital Campus,

Whitefield, Bangalore-560066.

Email: lokeshsdoc@gmail.com

Date of Submission: 12/07/2014.

Date of Peer Review: 14/07/2014.

Date of Acceptance: 23/07/2014.

Date of Publishing: 31/07/2014. 